Original Research Article

\title{
Estimation of platelet counts: auto analyzer versus counts from peripheral blood smear based on traditional and platelet: red blood cell ratio method
}

\author{
Balakrishnan A. ${ }^{1}$, Shetty A. ${ }^{2}$, Vijaya C. ${ }^{3}$ \\ ${ }^{1}$ Dr. Akhila Balakrishnan, 7th term MBBS Student, ${ }^{2}$ Dr. Archana Shetty, Associate Professor, Department of Pathology \\ ${ }^{3}$ Dr. Vijaya C, Professor and Head, Department of Pathology, Sapthagiri Institute of Medical Sciences and Research \\ Center Bangalore, Karnataka, India.
}

Corresponding Author: Dr. Archana Shetty, Associate Professor, Department of Pathology, Sapthagiri Institute of Medical Sciences and Research Center Bangalore. E-mail: archanashetty2924@gmail.com

\begin{abstract}
Introduction: Accurate platelet counts are of utmost importance in clinical practice The methods used for estimating platelet count are: Manual method using counting chamber, Examination of a peripheral blood smear (PBS) and automated hematology analyzers Automated hematology analyzers produce erroneous results in the presence of particles of similar size and/or light scatter like fragmented red blood cells (RBC), microcytic RBCs and in the presence of giant platelets and platelet clumps. Aims and Objectives: Comparison of platelet count by three methods: 1 . Automated six part analyzer. Traditional method: Counting average platelets per ten high power fields and multiplying the same by 15,000. 3. From smear by counting the number of platelets per $1000 \mathrm{RBCs}$ and calculating the platelet count on the basis of platelet: RBC ratio. Methods: Ethylene Diamine Tetra-acetic acid (EDTA) samples for platelet counts over a period of two months were analyzed by the above-mentioned methods. Statistical software SPSS 2 and independent T tests were used to compare the variables between the groups. Sensitivity and specificity of the methods was calculated. Results: 250 EDTA samples were analyzed of which normal platelet counts were $(56.4 \%)$ thrombocytopenic $(35.2 \%)$ and thrombocytosis $(8.4 \%)$. There was no significant difference between the platelet counts done by the auto analyzer compared with traditional (P value 0.50) and platelet: RBC ratio method (0.906). Specificity of the methods was $99.1 \%$ and the sensitivity was $92.5 \%$. Conclusion: Platelet counts by traditional and platelet: RBC ratio can be used as alternate reliable methods as compared to the auto analyzers.
\end{abstract}

Key words: platelet count, auto analyzers, peripheral smear, clumps, thrombocytopenia

\section{Introduction}

Platelets play a key role in homeostasis and thrombosis. Platelet count is one of the critical parameters in patient care, at times being a decisive factor for diagnosis and treatment. Platelet counts can be assessed by manual methods using counting chamber and examination of a peripheral blood smear (PBS). However, with the advent of automation, hematology analyzers have taken over in day to day practice from semi - automated to completely automated machines, based on the principles of impedance, flow cytometry and optical fluorescence.

Manual methods are time consuming, subjective and tedious with high levels of imprecision [1]. Automated hematology analyzers though rapid in giving results, at

Manuscript received: $10^{\text {th }}$ August 2018

Reviewed: $20^{\text {th }}$ August 2018

Author Corrected: $28^{\text {th }}$ August 2018

Accepted for Publication: $3^{\text {rd }}$ September 2018 times give erroneous values in the presence of giant platelets, platelet clumps, fragmented and microcytic red blood cells (RBCs) [2,3,4].

It is a standard protocol in most hematology laboratories, that abnormal platelet values generated by cell counters be followed by a manual examination of Leishman stained peripheral blood smear $[5,6,7]$. Also, in certain parts of the world where fully automated analyzers are not in use, alternate reliable methods can be considered. Studies have been documented in literature comparing the various manual and automated methods. The objective of this study is to compare the reproducibility of the platelet counts by the automated analyzer with counts obtained by traditional and platelet: red blood cell (RBC) ratio method using the same samples. 


\section{Original Research Article}

\section{Materials and Methods}

Place of study: Sapthagiri Institute Of Medical Sciences and Research Center.

Type of study: This prospective, descriptive, cross sectional study was conducted over a period of two months from May - June 2018. Institutional ethical committee approval was taken for the study.

Inclusion criteria: Anti-coagulated blood samples collected in Ethylene Diamine Tetra Acetic acid (EDTA) referred to the Central laboratory for platelet counts from subjects of all ages and both genders during the study period were included in the study. Exclusion criteria: Hemolysed and clotted samples were excluded from the study.

The samples were analyzed by three methods. Method 1: Samples were analyzed in automated six part hematology analyzer Sysmex XN550 using impedance method to get a complete blood count (CBC). The parameters run were standardized by routine external and internal quality control checks. Air dried thin smears were made from all samples and stained with
Leishman stain. These smears were then examined under Olympus $\mathrm{CH} 20$ i light microscope using 100x oil immersion lens. Junction of the body and tail of the smear, where the cells are monolayered was taken for counting. Two other methods were used for platelet counts. Method 2: The average number of platelets per ten high power fields was counted and this number was multiplied by 15,000 to get the platelet count in $10^{3} / \mu \mathrm{L}$ [8]. Method 3: Platelets were counted simultaneously with RBCs till a total of 1000 RBCs were counted. The number of platelets per $1000 \mathrm{RBCs}$ thus obtained was multiplied by the RBC count in $10^{6} / \mu \mathrm{L}$ to get an estimation of platelet count in $10^{3} / \mu \mathrm{L}$ [9]. The methods 2 and 3 were done independently by two observers and average values were taken to minimize inter-observer variation.

Statistical analysis: Data was entered in Excel sheet. Statistical software SPSS 2 and independent $\mathrm{T}$ tests were used to compare the variables between the groups. $\mathrm{P}$ value of $<0.05$ was considered significant. Sensitivity and specificity of the manual methods was compared with the autoanalyzer.

\section{Results}

A total of 250 samples were received in EDTA tubes for platelet counts during the study period. 121 were females and 129 were males. Mean age of the patients was 45.8 years.

Using automated method, the following were the categories of platelet counts among the 250 cases.

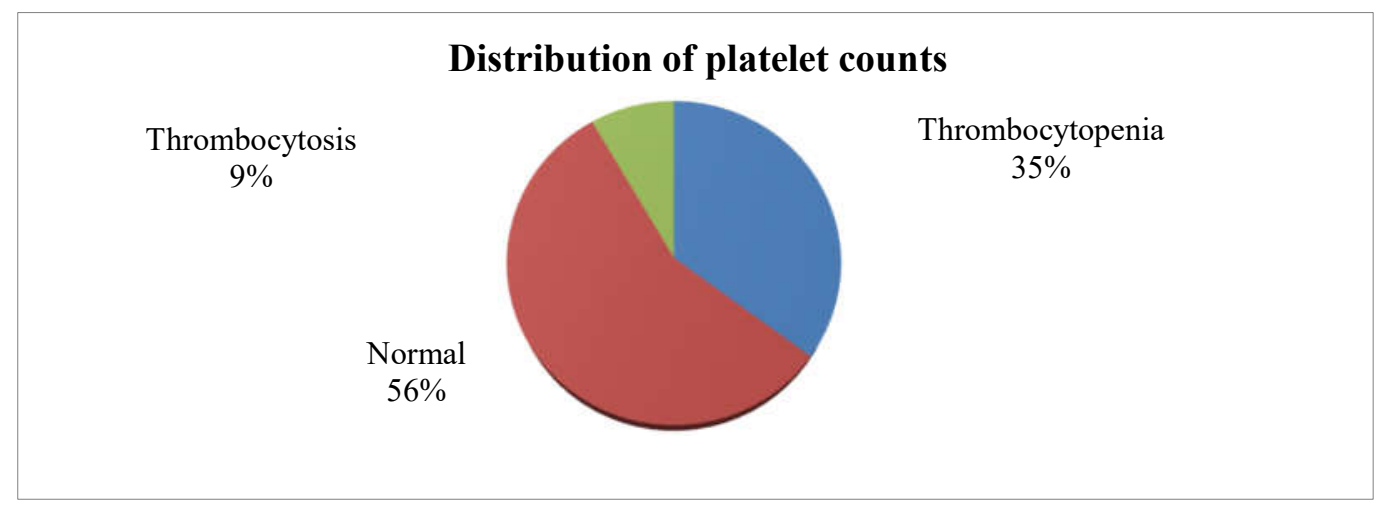

Graph-1: Distribution of platelet counts in the subjects (automated method)

The following were the reasons for getting platelet counts done as mentioned in the laboratory requisition forms: Preoperative checkups $(18.4 \%)$, routine checkups $(12 \%)$, health care schemes $(19.2 \%)$, dengue and other viral fevers $(25.2 \%)$, malaria $(15.6 \%)$ and patients on chemotherapy $(9.6 \%)$. No specific diseases or conditions were considered for exclusion from the study.

The mean platelet count by automated method was $224.58 \times 10^{3} / \mu \mathrm{L}$. The mean platelet count by the traditional method and platelet: RBC ratio method was $234.12 \times 10^{3} / \mu$ Land $226.08 \times 10^{3} / \mu \mathrm{L}$ respectively. The $\mathrm{P}$ value for automated method versus traditional method was 0.457 and for automated method versus platelet: RBC ratio method was 0.906 suggesting that there was no significant variation in the platelet counts between the manual methods when compared with the automated analyzer. 


\section{Original Research Article}

Table-1: Comparison between automated method and traditional method of platelet count.

\begin{tabular}{|c|c|c|c|c|c|}
\hline \multicolumn{2}{|c|}{} & Total cases & Mean & \multirow{2}{*}{ P-VALUE } \\
\hline & Automated method & 250 & 224.58 & & \\
\cline { 2 - 4 } & Traditional method & 250 & 234.12 & 0.572 & 0.457 \\
\hline
\end{tabular}

Table-2: Comparison between automated method and platelet: RBC ratio method of platelet count

\begin{tabular}{|l|l|l|l|l|l|}
\hline \multicolumn{2}{|l|}{} & Total cases & Mean & F & \multirow{2}{*}{ P-VALUE } \\
\hline \multirow{2}{*}{ Automated method } & 250 & 224.58 & & \\
\cline { 2 - 5 } & Platelet: RBC ratio method & 250 & 226.08 & 0.118 & 0.906 \\
\hline
\end{tabular}

Normally platelets are spread out single in the smears where the RBCs are monolayered as shown in figure below. Smears which showed falsely low platelet counts were found to have scattered platelet clumps or giant platelets as shown in the figures below.

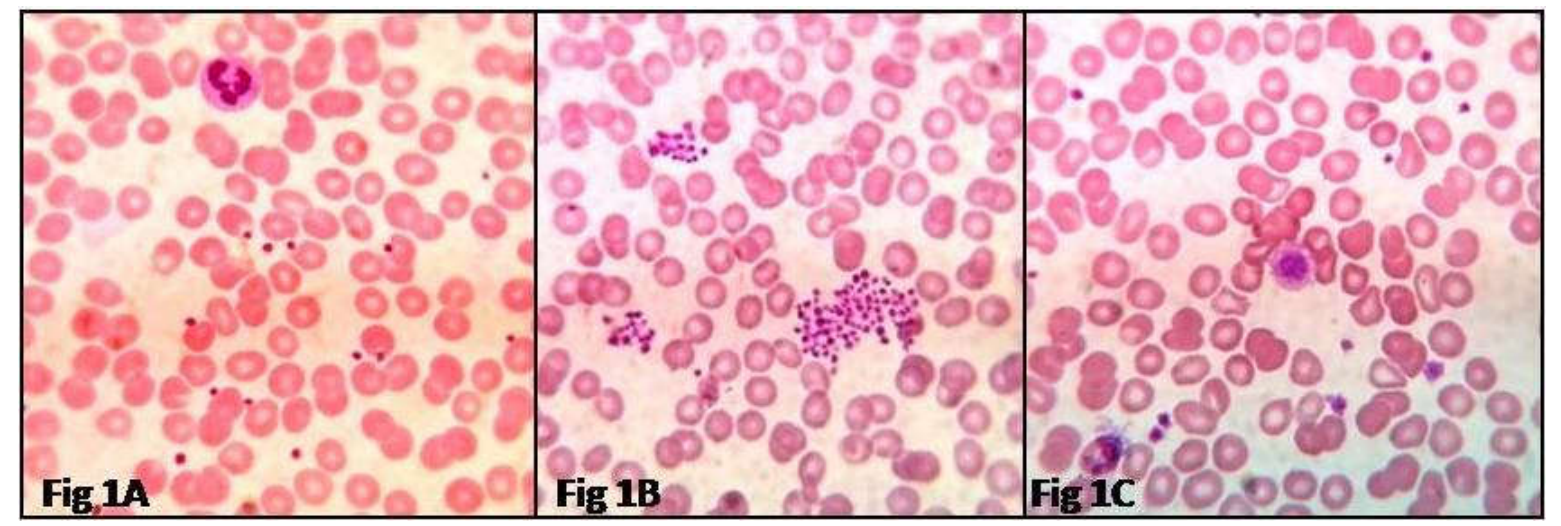

Figure-1A: Smear showing normal platelet distribution on a Leishman stained peripheral smear $x$ 100. Figure 1B: Smear showing platelet aggregates on smear Leishman stain $x$ 100. Figure 1C: Smear showing giant platelets on a Leishman stain x 100 .

Graphs were plotted to calculate the correlation between the methods as given below:

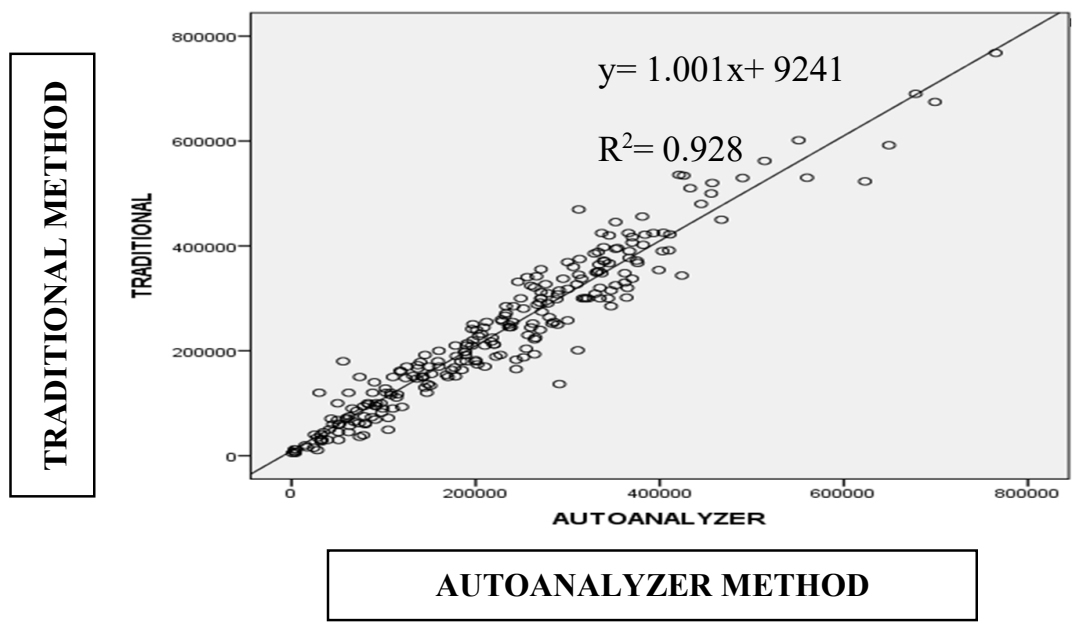

Graph 3: Scatter-plot showing correlation between automated method and traditional method.

$\mathrm{r}=96.3, \mathrm{p}=0.001$ The intra-class correlation co-efficient (ICC) was 0.963 indicating perfect positive correlation. 


\section{Original Research Article}
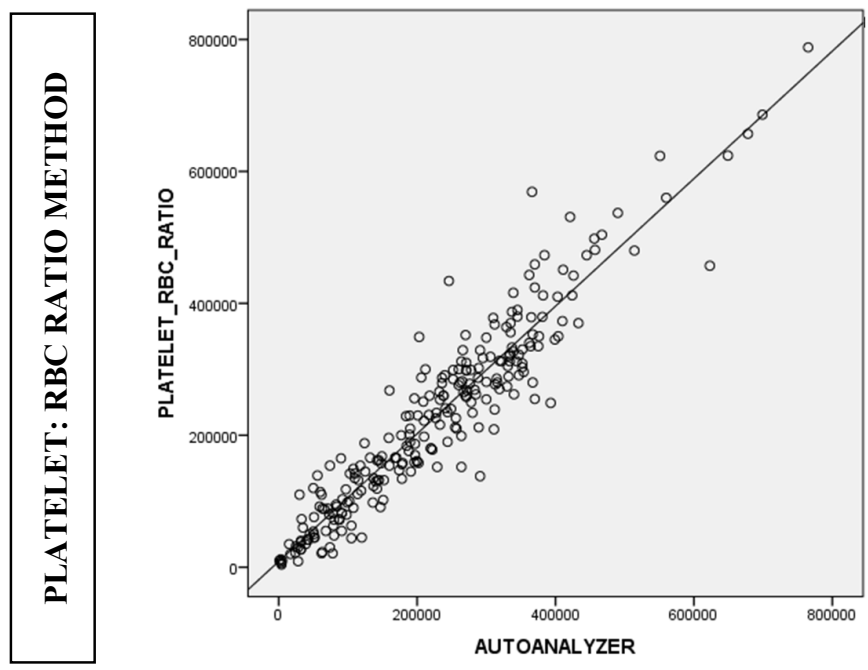

$y=0.967 x+8913.3$

$\mathrm{R}^{2}=0.893$

\section{AUTOANALYZER METHOD}

Graph-4: Scatter-plot showing correlation between automated method and platelet: RBC ratio method. $\mathrm{r}=94.1, \mathrm{p}=0.001$ The ICC was 0.941 indicating perfect positive correlation.

Sensitivity and specificity of traditional method and platelet: RBC ratio method were calculated in comparison to automated method. Normal platelet counts and thrombocytosis had been clubbed to calculate the same.

Table-3: Sensitivity and specificity of traditional method and platelet: RBC ratio method compared to automated method.

\begin{tabular}{|c|c|c|}
\hline & Traditional method & Platelet: RBC ratio method \\
\hline Sensitivity & $82.9 \%$ & $89.8 \%$ \\
\hline Specificity & $98.7 \%$ & $96.3 \%$ \\
\hline
\end{tabular}

The present study showed that there is no significant difference in the platelet counts obtained by the three methods.

\section{Discussion}

Giulio Bizzozero, an Italian pathologist is credited with identifying platelets as an important component of blood and their essential involvement in the mechanism of blood clotting at the end of the nineteenth century [10]. It is a well known fact that an accurate and reproducible platelet counts are of utmost importance in day-to-day clinical practice aiding not only in diagnosis but also in the treatment and prognostication of a wide variety of diseases [11]. Severe thrombocytopenia (platelet count $<150 \times 10^{3} / \mu \mathrm{L}$ ) can present as internal and external bleeding which maybe fatal and require emergency platelet transfusion. At the other extreme, thrombocytosis can cause thrombotic events [12].

In infections like malaria and dengue- with thrombocytopenic patients facing the potential risk of bleeding- an accurate count is imperative. Platelet count is also one of the parameters to assess the severity of dengue [13]. It is also important to prognosticate patients undergoing cytotoxic therapy for hematological malignancies. Studies have shown that platelet count is often reduced than normal in patients of Nonalcoholic fatty liver disease (NAFLD), thereby providing a supportive indicator in addition to liver biopsy in these patients. At present, platelet count is also used for prognostication of fibrosis and cirrhosis of the liver [14].

Various principles of doing automated platelet counts are impedance, optical scattering, optical fluorescence, and immunologic flow cytometry. The manual phase contrast microscopy method, although considered the gold standard was abandoned as it was time-consuming and not precise at low counts.

Immunological flow reference method also known as the RBC platelet ratio method replaced the manual phase contrast microscopy method as the international reference method (IRM) at the advent of the last 


\section{Original Research Article}

decade, which has led to greater precision in platelet counting and has resulted in its extensive use in laboratories worldwide. [15]. A few limitations of estimating platelet counts on Leishman stain smears are improper staining and stain artifacts. To overcome this, study by Uma Shankar et al [12] has shown that even the unstained peripheral smears prepared from fresh blood can be used to evaluate a platelet counts. However micro platelets are difficult to appreciate and large platelets resemble WBCs on unstained smears which must be considered [1]. A major factor contributing to the accuracy of platelet counts is the duration of sample storage post collection. The standard guidelines state that processing of hematological samples should be done within six to eight hours of collection, to avoid variations and errors in reporting the different hematological parameters $[16,17]$. A study by Jain et al has shown that platelet counts done on automated analyzers showed an unacceptable bias even within less than four hours of sample storage at 33.C for platelet counts [18]. In the present study, all the samples were run within a span of five hours from the time of collection.

Although hematology analyzers provide reliable full blood counts based on their linearity limits, they are known to be inaccurate and poorly reproducible at enumerating platelets in severe thrombocytopenia which maybe secondary to interference from cells or materials of a similar size to platelets or due to light scatter like in the case of fragmented RBCs, microcytic $\mathrm{RBCs}$, lipemic samples, debris in patients taking cytotoxic drugs or in the presence of giant platelets or platelet clumps. According to a study done by Barbara et al [15]. It was found that majority of the automated analyzers overestimated the platelet count especially when the levels were less than $200 \times 10^{3} / \mu \mathrm{L}$. Overestimation of thrombocytopenic platelet counts may result in the substantial under transfusion of platelets in high risk patients in need of platelet transfusion resulting in significant risk of under transfusion [15].

Therefore manual platelet estimation using various methods has been commonly used in settings of low platelet count for microscopically corroborating the auto analyzer counts. The evaluation of the peripheral blood smear till date still constitutes an indispensable tool in the evaluation of patients with hematologic disorders [19]. Mn Blood film evaluation offers added assessment of platelet size, shape, granulation, and can also be used to analyze platelet aggregation or satellitism. It is also essential for any standard of care test to be consistent, and a study carried out by AlHosni et al [11] demonstrated that manual platelet count estimation is reproducible in trained competent hands when a standardized methodology is used. In our study the manual methods were done independently by two observers and average values obtained were used so as to keep interobserver bias to the minimum. An average of interobserver CVs in the range of $10-25 \%$ has been reported in previous studies as against CVs of less than $3 \%$ for automated blood counts [20]. A few studies have suggested the standardization of the manual platelet count methods in relation to the microscopes used for doing the counts, the region of the smears examined on the slides and the laboratory personnel involved. Also proposals that determination of a correction factor be performed in each laboratory in order to improve the accuracy and reliability of platelet estimates [21].

The present study has shown that manual methods are reliable to validate the automated counts in routine practice under standard conditions. In addition essential that pathology laboratory personals and clinicians who rely on platelet counts for various scenarios in day to day practice understand the limitations of the instrumentation in use and the measurement uncertainty of automated platelet counters.

However, limitations of the study are a small sample size and that reproducibility of the manual methods in case of thrombocytopenia, thrombocytosis and normal ranges have not been studied independently. Other aspects that could affect the duplicability of these methods like platelet volume, underlying disease, method used to prepare the blood film have also not been taken into consideration in our study. Larger sample studies with more stringent criteria are recommended to throw light on the same.

\section{Conclusion}

Our study tries to validate the manual methods for use in circumstances wherein a fully automated hematology analyzer is not available as is the case usually in primary health care centers or urban health centers .This study comes into importance in a developing country like India, especially in semi-urban/rural areas where possibly only semi-automated hematology analyzers are available.

This study reaffirms that manual methods may also be used to validate the analyzers and in rare situations when the fully automated analyzers shut down and immediate patient care may essentially be based on the platelet counts alone.

Pathology Update: Tropical Journal of Pathology \& Microbiology Available online at: www.pathologyreview.in 393 | P a g e 


\section{Original Research Article}

Acknowledgement: We thank Dr. Kruthika and Mrs. Lavanya from the department of community medicine for helping with the statistical analysis of the study.

Contribution by individual authors

\begin{tabular}{|c|c|c|c|}
\hline Nature of work Authors & Author 1 & Author 2 & Author 3 \\
\hline Concepts & Yes & Yes & Yes \\
\hline Design & & Yes & \\
\hline Definition of intellectual content & & Yes & \\
\hline Literature search & Yes & Yes & \\
\hline Data acquisition & Yes & Yes & \\
\hline Data analysis & Yes & Yes & Yes \\
\hline Statistical analysis & Yes & Yes & Yes \\
\hline Manuscript preparation & Yes & Yes & \\
\hline Manuscript editing & Yes & Yes &
\end{tabular}

Funding: Nil, Conflict of interest: None initiated

Permission from IRB: Yes

\section{References}

1. Briggs $\mathrm{C}$, Harrison $\mathrm{P}$, Machin SJ. Continuing developments with the automated platelet count. Int LabHem 2007;29:77-91. 10.5958/2394-6792.2016. 00066.1

2. Boulassel M-R, Al-Farsi R, Al-Hashmi S, Al-Riyami $\mathrm{H}$, Khan H, Al-KindiS. Accuracy of platelet counting by optical and impedance methods in patients with thrombocytopenia and microcytosis. Sultan Qaboos Univ Med J. 2015;15(4):e463-8. doi: 10.18295/squmj.2015.15.04.004

3. Marionneaux S, Francisco N, Chan V, Hanenberg J, Rafael J, Chua C, et al. Comparison of automated platelet counts and potential effect on transfusion decisions in cancer patients. Am J Clin Pathol. 2013; 140 (5): 747-54.10. 1309/ AJCP58 INTIT VGQZI

4. Brahimi M, Osmani S, Arabi A, et al. The estimation of platelet count from a blood smear on the basis of the red cell: platelet ratio. Turk J Heamtol2009;26 (1):21-4

5. Hutchinson RE, Mc Pherson RA, Schexneider KI. Basic examination of blood and bone marrow. In: Mc Pherson RA, Pincus MR, editors. Henry's clinical diagnosis and management by laboratory methods.22nd ed. Philadelphia:Elsevier Saunders; 2011: 509-35.

6. Moreno A, Menke D. Assessment of platelet numbers and morphology in the peripheral blood smear. Clin Lab Med.2002;22(1):194-213
7. Rodak BF, Fritsma GA, Doig K. Hematology: Clinical principles and applications. 4th ed. Elsevier- Saunders; 2011; p. 202.

8. Malok M, Titchener EH, Bridgers C, Lee BY, Bamberg $\mathrm{R}$ Comparison of two platelet count estimation methodologies for peripheral blood smears. Clin Lab Sci. 2007 summer; 20 (3): 154-60.

9. UmaraniMK, Shashidhar HB. Estimation of platelet count from peripheral blood smear based on platelet: red blood cell ratio. A prospective study in a tertiary care hospital. Indian Journal of Pathology and Oncology, April-June 2016;3(2):351-353.10.5958/ 2394-6792. 2016.00066.1

10. Balduini, C. L.\&Noris, P. Platelet count and aging. Haematologica 2014; 99: 953-955.

11. Al-Hosni ZS, Al-Khabori M, Al-Mamari S, et al. Reproducibility of Manual Platelet Estimation Following Automated Low Platelet Counts. Oman Medical Journal. 2016; 31(6):409-413. 10.5001/omj. 2016.83

12. Umashankar T, Thomas BM, Sahana P Estimation of platelet count in unstained peripheral blood smears in comparison with stained smears and evaluation of its efficacy. Estimation of platelet count in unstained peripheral blood smears in comparison with stained smears and evaluation of its efficacy. Malays J Pathol. 2014 Dec; 36(3): 195-9. 


\section{Original Research Article}

13. Phakhounthong $\mathrm{K}$, Chaovalit $\mathrm{P}$, Jittamala $\mathrm{P}$, BlacksellSD, Carter MJ, Turner $\mathrm{P}$ et al. Predicting the severity of dengue fever in children on admission based on clinical features and laboratory indicators: application of classification tree analysis. BMC Pediatrics. 2018; 18:109.doi:10. 1186/s 12887-018-1078-y.

14. MilovanovicAlempijevic T, StojkovicLalosevic M, Dumic I, et al. Diagnostic Accuracy of Platelet Count and Platelet Indices in Noninvasive Assessment of Fibrosis in Nonalcoholic Fatty Liver Disease Patients. Canadian Can J Gastroenterol Hepatol. 2017; 2017: 6070135. doi:10.1155/2017/ 6070135 .

15. De la Salle BJ, McTaggart PN, Briggs C, Harrison $\mathrm{P}$, Doré CJ, Longair I, et al. The accuracy of platelet counting in thrombocytopenic blood samples distributed by the UK National External Quality Assessment Scheme for General Haematology. Am J Clin Pathol. 2012 Jan;137 (1):65-74.doi.org/10. 1309/AJCP86JMBFUCFCXA

16. Buttarello M. Quality specification in hematology: the automated blood cell count. Clin Chim Acta. 2004;346:45-54. doi: 10.1016/j.cccn.2004.02.03
17. National Accreditation Board for Testing and Calibration Laboratories (IN). NABL 112 Specific Criteria for Accreditation of Medical Laboratories. Gurgaon: National Accreditation Board for Testing and Calibration Laboratories; 2016. p. 36

18. Jain A, Jain S, Singh N, et al. Storage stability of commonly used hematological parameters at $33{ }^{\circ} \mathrm{C}$ : Electronic supplementary material available online for this article. Biochem Med (Zagreb). 2018 Jun 15; 28(2): 020901

19. Bain BJ. Diagnosis from the blood smear. N Engl J Med. 2005;353:498-507 DOI: 10.1056/NEJMra 043442

20. Dadu T, Sehgal K, Shaikh A, Khodaiji S. Comparison of platelet counts by sysmex XE 2100 and LH-750 with the international flow reference method in thrombocytopenic patients. Indian $\mathrm{J}$ Pathol Microbiol. 2013;56: 114-119DOI: 10. 4103/ 0377-4929.118701

21. Comar SR, Danchura HSM, Silva PH. Platelet count: evaluation of manual methodologies and application in the laboratory routine. Rev Bras Hematol Hemoter. 2009;31(6):1-6. http://dx.doi. org/ 10. 1590/S1516-84842009005000087.

\section{How to cite this article?}

Balakrishnan A, Shetty A, Vijaya C. Estimation of platelet counts: auto analyzer versus counts from peripheral blood smear based on traditional and platelet: red blood cell ratio method. Trop J Path Micro 2018; 4(5):389-395. doi:10.17511/jopm.2018.i05.04 